\title{
Atividades funcionais e cotidianas relacionadas com o desempenho cognitivo de nonagenários
}

\author{
Bruna Borba Neves; ;osemara de Paula Rocha*;:Marlon Cássio Pereira Grigol ${ }^{\star \star *}$; Gabriela Guimarães \\ Oliveira $^{\star \star \star *}$; Luisa Braga Jorge ${ }^{\star \star \star * *} ;$ Ângelo José Gonçalves Bós ${ }^{\star \star \star \star * \star *}$
}

\section{Resumo}

O objetivo do estudo foi investigar a relação entre cognição e facilidade em desempenhar atividades cotidianas e funcionais entre nonagenários e o impacto das atividades investigadas sobre a cognição, ajustando para sexo, idade, escolaridade e sintomas depressivos. Trata-se de estudo transversal e analítico. Foram selecionados nonagenários residentes em Porto Alegre, RS em 2016, através de amostragem por conglomerados. Foram variáveis do estudo: a cognição (Mini-Exame do Estado Mental - MEEM), variáveis sociodemográficas (sexo, escolaridade e faixa etária), sintomas depressivos (escala de depressão geriátrica - GDS) e o grau de facilidade em executar 12 atividades funcionais e cotidianas. O grau de facilidade na realização de cada uma das atividades teve sua relação com o MEEM testada pelo teste de qui-quadrado, ANOVA e regressão linear simples ajustada para as características sociodemográficas e clínicas. Participaram 208 nonagenários, 71\% mulheres. Tanto na análise simples quanto na análise ajustada, maior facilidade na execução de cada uma das atividades foi relacionada significativamente com maiores escores no MEEM. Sexo masculino, mais que 5 anos de estudo, menores sintomas depressivos e menos que 95 anos de idade foram variáveis relacionadas com maiores relatos de facilidade para realização das atividades

" Terapeuta Ocupacional. Mestrado e doutoranda em Gerontologia Biomédica pela Pontifícia Universidade Católica do Rio Grande do Sul, Porto Alegre/RS. Especialista em Terapia Ocupacional em Gerontologia pelo Conselho Federal de Fisioterapia e Terapia Ocupacional e em Saúde Pública pelo Centro Universitário Cidade Verde, Maringá/PR. E-mail: brunanevesto@hotmail.com.

** Fisioterapeuta. Doutoranda em Gerontologia Biomédica pela Pontifícia Universidade Católica do Rio Grande do Sul, Porto Alegre/RS. Mestrado em Envelhecimento Humano pela Universidade de Passo Fundo, Passo Fundo/RS. E-mail: rocha.josemara.paula@gmail.com.

*** Fisioterapeuta. Mestrado em Gerontologia Biomédica pela Pontifícia Universidade Católica do Rio Grande do Sul, Porto Alegre/RS. E-mail: marlonfisio@gmail.com

****** Fisioterapeuta. Mestrado e doutoranda em Gerontologia Biomédica pela Pontifícia Universidade Católica do Rio Grande do Sul, Porto Alegre/RS. Especialização em Gestão da Atenção à Saúde do Idoso pelo Centro de Educação Tecnológica e Pesquisa em Saúde do Grupo Hospitalar Conceição/Instituto Federal de Educação, Ciência e Tecnologia do Rio Grande do Sul, Porto Alegre/RS. E-mail: oliveira gabriela@hotmail.com.

***** Fisioterapeuta. Mestrado e doutoranda em Gerontologia Biomédica pela Pontifícia Universidade Católica do Rio Grande do Sul, Porto Alegre/RS. Especialização em Fisioterapia Pélvica/Uroginecológica pela INSPIRAR, Curitiba/PR. E-mail: lbragajorge@hotmail.com.

******* Médico. Professor titular da Escola de Medicina e pesquisador do Instituto de Geriatria e Gerontologia da Pontifícia Universidade Católica do Rio Grande do Sul, Porto Alegre/RS. Doutorado em Phd In Medicine com ênfase em Saúde Comunitária pela Tokai University, Japão. Pós-doutorado sobre Envelhecimento no Instituto Nacional Americano e na Escola de Saúde Pública da Universidade de Johns Hopkins em Baltimore, EUA. E-mail: angelo.bos@pucrs.br

hhttp://dx.doi.org//10.5335/rbceh.v16i3.7608 
cotidianas e funcionais. As atividades relacionadas ao auto-cuidado apresentaram os maiores coeficientes de regressão para os escores do MEEM. Os achados sugerem que a facilidade em desempenhar atividades funcionais e cotidianas estão associadas com escores mais altos no MEEM em nonagenários, independente da escolaridade, sintomas depressivos, sexo e faixa etária.

Palavras-chave: Longevidade. Envelhecimento. Demência. Atividades cotidianas. Cognição.

\section{Introdução}

O envelhecimento é um processo dinâmico, progressivo e irreversível. Suas causas vêm sendo estudadas por teorias de diversos embasamentos científicos, aceitando-se estar determinado a fatores biológicos, psíquicos e sociais (FECHINE; TROMPIERI, 2012). No processo de envelhecimento as alterações cognitivas são frequentemente confundidas como um processo natural da idade cronológica, porém esse tipo de raciocínio pode retardar o diagnóstico precoce e o tratamento de patologias que comprometem a capacidade cognitiva, principalmente em pessoas com idade mais avançada (longevos). A detecção precoce do declínio cognitivo em idosos é uma estratégia importante na tentativa de reduzir a velocidade do agravamento cognitivo esperado e para prevenir danos secundários (NASCIMENTO et al., 2015).

A função cognitiva é determinante para a manutenção de uma vida independente, sendo a sua perda considerada um marcador de declínio e fragilidade física e funcional, interferindo na realização de atividades cotidianas. Portanto, o prejuízo encontrado na dimensão da capacidade funcional pode ser justificado pelo grau de habilidades cognitivas (CHAVES et al., 2015).

O envelhecimento tem sido acompanhado do declínio em algumas habilidades cognitivas, aumentando, portanto, a ocorrência das doenças psiquiátricas, entre as quais a demência e a depressão se destacam como os transtornos mentais mais comuns em idosos (GOYANNA et al., 2017). No cotidiano do idoso as atividades de vida diária (AVD) se enquadram no complexo conjunto de competências comportamentais associadas à funcionalidade, à motivação e a experiências anteriores, dependendo da preservação das funções físicas e cognitivas. Entretanto, as alterações nas condições clínicas de saúde que cursam com o envelhecimento frequentemente prejudicam o engajamento em AVD, que englobam atividades com diferentes graus de complexidade (SPOSITO; NERI; YASSUDA, 2016).

Os longevos e principalmente os centenários são considerados por alguns autores, como sobreviventes, acreditando possuírem características protetoras que os diferenciam de outros grupos etários mais jovens (CÔRRO, 2016). Em se tratando de nonagenários brasileiros pesquisas específicas com essa população podem ajudar a entender se essas características diferenciais realmente existem e quais são fatores importantes a serem promovidos na busca de promoção à saúde nessa faixa etária. Dentre os 
determinantes cognitivos conhecidos na literatura, um estudo longitudinal investigou determinantes do declínio cognitivo de idosos e demonstrou que o baixo nível de anos de estudo, gênero e idade se associou com o declínio cognitivo em 10 anos (CASTRO-COSTA et al., 2011). A depressão também pode ser considerada um fator de risco para a demência, todavia, não se sabe ainda em que momento do processo de envelhecimento ela possa ser determinante para o surgimento de demência (LIVINGSTON et al., 2017).

Estudos longitudinais referem associação significativa entre o desempenho das AVD e o desempenho cognitivo. Dias et al. (2015) avaliaram o impacto das AVD na incidência de declínio cognitivo e observaram que um desempenho satisfatório em AVD mostra ser um fator protetor para o desenvolvimento de declínio cognitivo, independentemente de condições socioeconômicas, de saúde geral e estilo de vida. Da mesma forma, Figueiredo et al. (2013) ao realizarem um acompanhamento de seis meses com idosos da comunidade encontraram resultados semelhantes. Ao correlacionarem a pontuação total do MEEM com as pontuações das AVD, em cada tempo do estudo, observou-se que os indivíduos com melhor desempenho cognitivo eram mais independentes nas AVD, enfatizando que a participação em atividades pode trazer benefícios para a cognição.

No entanto, não há um consenso sobre o mecanismo pelo qual as AVD atuam como protetoras do declínio cognitivo. Porém, sugere-se que os padrões de atividade e as redes neurais relacio- nadas que são estabelecidos no início da vida possam ser mais importantes para manter a saúde cognitiva na velhice, no sentido de minimizar os efeitos da diminuição da velocidade de processamento cognitivo no envelhecimento. (DIAS et al., 2015; FIGUEIREDO et al., 2013).

O objetivo desse estudo, nesse cenário, foi investigar se há relação entre cognição e facilidade em desempenhar atividades cotidianas e funcionais entre nonagenários e o impacto das atividades investigadas sobre a cognição, ajustando para determinantes cognitivos (sexo, idade, escolaridade e sintomas depressivos).

\section{Materiais e métodos}

Foram selecionados idosos participantes do Projeto de Atenção Multiprofissional ao Longevo (AMPAL) que é um estudo longitudinal realizado pela Pontifícia Universidade Católica do Rio Grande do Sul (PUCRS). A técnica de amostragem para criação da amostra do AMPAL foi de conglomerados representativos dos bairros de Porto Alegre, capital do Rio Grande do Sul. A cidade de Porto Alegre está dividida em setores censitários através do sistema disponibilizado pelo ObservaPOA (2015). No site foi possível identificar os setores que tinham pelo menos seis longevos. Após a identificação criou-se uma lista de longevos distribuídos por setores, sendo a partir disso, sorteados para participar da pesquisa. Em seguida, foi realizada uma busca ativa dos possíveis longevos em seus domicíclio e por meio de ligação telefônica, agendadas as avaliações de 
acordo com 0 aceite. Nas visitas eram apresentados e assinados os termos de consentimento livre e esclarecido para confirmar a aceitação.

Os critérios para participação era ser idoso de 90 anos ou mais, domiciliados em Porto Alegre e que não residiam em instituições de longa permanência, sendo incluídos idosos com qualquer nível funcional. Foram excluídos nonagenários cujos formulários apresentavam informações incompletas. O projeto obedeceu aos critérios éticos referentes aos pacientes e aceitos pelo comitê de ética e pesquisa da PUCRS sendo aprovado sob o parecer consubstanciado de número 1.639.961/2016, CAEE 59906216.0.0000.5336. Os nonagenários foram visitados no período de Janeiro a Outubro de 2016, as avaliações foram realizadas por meio de um questionário sociodemográfico elaborado no estudo AMPAL, o MEEM, a GDS e o grau de facilidade e dificuldade em realizar 12 atividades funcionais e cotidianas.

A variável dependente do estudo foi o escore do MEEM, e as variáveis independentes do estudo foram sociodemográficas: o sexo, a escolaridade $(<$ 5 e $\geq 5$ anos), a faixa etária $(<95$ anos e $\geq 95$ anos), e clínicas (GDS), e o grau de facilidade em executar 12 atividades funcionais e cotidianas. A escolha das atividades analisadas foi desenvolvida a partir de uma discussão com a equipe multiprofissional de pesquisadores do AMPAL e algumas atividades funcionais foram adaptadas do estudo de SIMONSICK et al. (2001). As atividades funcionais eram: caminhar $400 \mathrm{~m}$, subir dez degraus, carregar cinco quilos, levantar da cadeira sem apoio, agachar-se, levantar os braços acima do nível da cabeça e agarrar objetos com as mãos. As atividades cotidianas foram: transferir-se entre assentos, tomar banho sozinho, vestir-se sozinho, alimentar-se sozinho e usar o banheiro. Os entrevistados eram questionados quanto ao grau de facilidade de desempenhar essas atividades citadas (autopercepção de esforço), atribuindo nota 0 se não conseguisse, 1 difícil, 2 mais ou menos fácil, e 3 fácil. Para fins de análise estatística foram agrupadas as categorias difícil e não consegue como "Difícil" e as categorias mais ou menos fácil e fácil como "Fácil".

A pontuação do MEEM varia de 0 a 30 pontos e envolve dez questões pertinentes a orientação temporal e espacial, três relacionadas a memória imediata, cinco à atenção e cálculo, três à evocação (memória evocativa), e dez correspondentes a área de linguagem (FOLSTEIN; FOLSTEIN; MCHUGH, 1975). No presente trabalho o MEEM foi tratado como variável numérica. Para análise dos sintomas depressivos foi utilizado o GDS de cinco questões proposto por Almeida (2010). A autora também sugere como ponto de corte $<2$ questões positivas para sintoma depressivo como "Ausência de depressão" e $\geq 2$ questões como "Presença de depressão".

Os dados foram analisados pelo pacote estatístico Epi Info versão 7.2. Para observar a possível diferença nas médias do MEEM entre os níveis de sexo, escolaridade, faixa etária e presença de sintomas de depressão foi utilizado o 
teste de análise de variância (ANOVA). A possível relação da distribuição das variáveis independentes citadas anteriormente e o grau de facilidade na execução das atividades funcionais e cotidianas foi testada pelo Qui-quadrado assim como comparadas as médias de MEEM para as atividades, sendo essas também testadas pelo teste de ANOVA. As análises da distribuição das atividades e os outros fatores sociodemográficos e clínicos foram realizadas para observar a possível necessidade de análise ajustada para as mesmas quando os fatores fossem significativos tanto para o MEEM quanto para as atividades (potenciais fatores de confusão entre MEEM e facilidade para executar as atividades). Regressões lineares para identificar quais atividades do cotidiano estavam relacionadas com o escore do MEEM ajustando para potenciais fatores de confusão foram realizadas. A permanência de correlação significativa após o ajuste confirmaria o grau de associação entre MEEM e a facilidade em executar as atividades funcionais e cotidianas independente dos outros fatores. Valores de $\mathrm{p}<0,05$ foram considerados estatisticamente significativos.

\section{Resultados}

Participaram 208 nonagenários, sendo a maioria do sexo feminino 148 (71\%). Em relação ao MEEM, a maior média foi encontrada em relação aos homens (23,3 $\pm 5,79$ ) sendo significativamente maior que a das mulheres $(20,1 \pm 6,60 ; p<$
0,001), indicando uma maior preservação do estado cognitivo nos homens.

O maior percentual de participantes foi encontrado com $<5$ anos de estudo (54\%), grupo este com menor pontuação no $\operatorname{MEEM}(18,8 \pm 6,16 ; \mathrm{p}<0,001)$. A maioria da amostra (80\%) tinha menos que 95 anos e apresentavam maior média no $\operatorname{MEEM}(21,8 \pm 6,36)$ quando comparado a amostra com maior de $95(17,9 \pm 6,36)$. Então quanto menor a idade maior a pontuação no MEEM, sendo essa relação significativa $(p<0,001)$.

$\mathrm{Na}$ GDS foi encontrado na população analisada $55 \%$ dos idosos com menos de dois sintomas depressivos e $45 \%$ com dois ou mais sintomas de depressão, revelando uma relação significativa ( $\mathrm{p}$ $<0,001$ ), sendo que, menor a pontuação no GDS, maior a pontuação no MEEM. As características de distribuição dos idosos quanto aos dados sociodemográficos, clínicos e a média obtida nos escores do Mini Exame do Estado Mental estão apresentados na Tabela 1 . 
Tabela 1 - Distribuição dos participantes quanto às características sociodemográficas e clínicas e a média obtida nos escores do MEEM, Porto Alegre/RS, Brasil, $2019(n=208)$.

\begin{tabular}{|c|c|c|c|}
\hline Variáveis/categorias & n (\%) & $\begin{array}{l}\text { MEEM } \\
\text { (Média } \pm \\
\text { DP) }\end{array}$ & $\mathbf{p}$ \\
\hline \multicolumn{4}{|l|}{ Sexo } \\
\hline Mulheres & $148(71)$ & $20,1 \pm 6,60$ & \multirow[t]{2}{*}{$<0,001$. } \\
\hline Homens & $60(29)$ & $23,3 \pm 5,79$ & \\
\hline \multicolumn{4}{|l|}{ Escolaridade } \\
\hline$<5$ anos & $113(54)$ & $18,8 \pm 6,16$ & \multirow[t]{2}{*}{$<0,001$} \\
\hline$\geq 5$ anos & $95(46)$ & $23,5 \pm 6,05$ & \\
\hline \multicolumn{4}{|l|}{ Faixa etária } \\
\hline$<95$ anos & $166(80)$ & $21,8 \pm 6,36$ & \multirow[t]{2}{*}{$<0,001$} \\
\hline$\geq 95$ anos & $42(20)$ & $17,9 \pm 6,36$ & \\
\hline \multicolumn{4}{|l|}{ GDS } \\
\hline$<2$ sintomas & $115(55)$ & $22,3 \pm 6,01$ & \multirow[t]{2}{*}{$<0,001$} \\
\hline$\geq 2$ sintomas & $93(45)$ & $19,4 \pm 6,85$ & \\
\hline
\end{tabular}

Nota: DP = Desvio-padrão; GDS = Geriatric Depression Scale.

Na Tabela 2 se observam as frequências dos fatores sociodemográficos e as médias dos fatores clínicos (MEEM e GDS) entre o grau de facilidade e dificuldade no desempenho das atividades funcionais. As médias de MEEM e GDS foram estatisticamente diferentes em todas as atividades. A variável sexo apresentou significância entre quase todas as atividades funcionais, exceto "agarrar objetos com as mãos". Faixa etária e Anos de estudo foram significativos com caminhar 400 metros, subir dez degraus, levantar-se da cadeira e carregar cinco quilos. Anos de estudo foi significativo também com agachar-se e agarrar objetos com as mãos.

Os homens apresentaram maior relato de facilidade em realizar as atividades, quando comparado com as mulheres, principalmente nas atividades de caminhar, subir dez degraus, carregar peso e levantar da cadeira sem apoio. Em relação à faixa etária, a principal diferença está na atividade de subir dez degraus, que os idosos $\geq 95$ anos relataram maior dificuldade em comparação com os de idade menor. Os indivíduos com $\geq 5$ anos de estudo relataram maiores percentuais de facilidade para caminhar, subir, carregar e levantar da cadeira sem apoio em relação aos que tinham $<5$ anos de estudo. Os indivíduos que tinham dificuldade em realizar as atividades funcionais apresentavam maior média no GDS e menor pontuação no MEEM. 
Tabela 2 - Diferenças no desempenho das atividades funcionais entre diferentes níveis de fatores sociodemográficos e clínicos, Porto Alegre/RS, Brasil, 2019 ( $n=2$ 08).

\begin{tabular}{|c|c|c|c|c|c|c|c|c|c|}
\hline \multirow{2}{*}{$\begin{array}{c}\text { Variá- } \\
\text { veis }\end{array}$} & \multicolumn{2}{|c|}{ Sexo§ } & \multicolumn{2}{|c|}{ Faixa Etária§ } & \multicolumn{2}{|c|}{ Anos de estudo§ } & \multirow{2}{*}{$\begin{array}{c}\text { MEEM£ } \\
\begin{array}{c}\text { Média } \pm \\
\text { DP }\end{array}\end{array}$} & \multirow{2}{*}{$\begin{array}{c}\text { GDSE } \\
\text { Média } \\
\pm \text { DP }\end{array}$} & \multirow{2}{*}{$\frac{\text { Total }}{\text { n (\%) }}$} \\
\hline & F n (\%) & $\begin{array}{c}\text { M } \\
\%(\end{array}$ & $<95$ & $\geq 95$ & $<5$ & $\geq \mathbf{5}$ & & & \\
\hline \multicolumn{10}{|c|}{ Caminhar 400m } \\
\hline Difícil & $92(62)^{\star \star}$ & $\begin{array}{l}25 \\
(42)\end{array}$ & \begin{tabular}{|l}
84 \\
$(51)$
\end{tabular} & $33(79)^{\star \star}$ & $71(63)$ & $46(48)^{\star \star}$ & $\begin{array}{c}19,4 \pm \\
6,69^{\star \star}\end{array}$ & $\begin{array}{c}1,79 \pm \\
1,19^{\star *}\end{array}$ & $117(57)$ \\
\hline Fácil & $56(38)$ & $\begin{array}{lr}3 & 5 \\
(58)\end{array}$ & $82(49)$ & $9(21)$ & $42(37)$ & $49(52)$ & $\underset{5,73}{23,0} \pm$ & $\begin{array}{c}1,14 \\
0,97\end{array}$ & $91(43)$ \\
\hline \multicolumn{10}{|c|}{ Subir dez degraus } \\
\hline Difícil & $84(57)^{\star}$ & \begin{tabular}{|l}
22 \\
$(37)$ \\
\end{tabular} & $\begin{array}{l}77 \\
(46) \\
\end{array}$ & $29(69)^{\star \star}$ & $71(63)$ & $35(37)^{\star \star}$ & $\begin{array}{c}18,70 \pm \\
6,59^{\star *}\end{array}$ & $\begin{array}{c}1,75 \pm \\
1,23^{\star *}\end{array}$ & $106(53)$ \\
\hline Fácil & $64(43)$ & $\begin{array}{l}38 \\
(63)\end{array}$ & $\begin{array}{l}89 \\
(54)\end{array}$ & $13(31)$ & $42(37)$ & $60(63)$ & $\underset{5,57}{23,4} \pm$ & $\begin{array}{c}1,2 \quad 5 \pm \\
0,99\end{array}$ & $102(47)$ \\
\hline \multicolumn{10}{|c|}{ Carregar cinco quilos } \\
\hline Difícil & $\begin{array}{l}101 \\
(68)^{\star \star}\end{array}$ & \begin{tabular}{|l}
21 \\
$(35)$ \\
\end{tabular} & \begin{tabular}{|l}
90 \\
$(54)$ \\
\end{tabular} & $32(76)^{\star \star}$ & $76(67)$ & $46(48)^{\star \star}$ & $\begin{array}{c}19,2 \pm \\
6,55^{\star \star}\end{array}$ & $\begin{array}{c}1,77 \pm \\
1,17^{\star \star}\end{array}$ & $122(61)$ \\
\hline Fácil & $47(32)$ & $\begin{array}{l}39 \\
(65)\end{array}$ & $\begin{array}{l}76 \\
(46)\end{array}$ & $10(24)$ & $37(33)$ & $49(52)$ & $\underset{5,65}{23,5} \pm$ & $\begin{array}{c}1,13 \\
0,99\end{array}$ & $86(39)$ \\
\hline \multicolumn{10}{|c|}{ Levantar da cadeira sem apoio } \\
\hline Difícil & $90(61)^{\star}$ & \begin{tabular}{|l}
28 \\
$(47)$
\end{tabular} & \begin{tabular}{|l}
83 \\
$(50)$
\end{tabular} & $35(83)^{\star \star}$ & $73(65)$ & $45(47)^{\star \star}$ & $\begin{array}{c}19,3 \pm \\
6,96^{\star \star}\end{array}$ & $\begin{array}{c}1,65 \pm \\
1,21^{\star *}\end{array}$ & $118(58)$ \\
\hline Fácil & $58(39)$ & \begin{tabular}{|l}
32 \\
$(53)$
\end{tabular} & \begin{tabular}{|l}
83 \\
$(50)$
\end{tabular} & $7(17)$ & $40(35)$ & $50(53)$ & $\underset{5,22}{23,2} \pm$ & $\underset{1,02}{1,32} \pm$ & $90(42)$ \\
\hline \multicolumn{10}{|c|}{ Agachar-se } \\
\hline Difícil & $65(44)^{\star \star}$ & $\begin{array}{l}18 \\
(30)\end{array}$ & $\begin{array}{l}65 \\
(39)\end{array}$ & $18(43)$ & $53(47)$ & $30(32)^{\star *}$ & $\begin{array}{c}19,1 \pm \\
6,78^{\star \star}\end{array}$ & $\begin{array}{c}1,85 \pm \\
1,22^{\star *}\end{array}$ & $83(42)$ \\
\hline Fácil & $83(56)$ & $\begin{array}{l}42 \\
(70)\end{array}$ & $\begin{array}{l}101 \\
(61)\end{array}$ & $24(57)$ & $60(53)$ & $65(68)$ & $\underset{6,06}{22,3} \pm$ & $\underset{1,03}{1,28} \pm$ & $125(58)$ \\
\hline \multicolumn{10}{|c|}{ Levantar os braços acima do nível da cabeça } \\
\hline Difícil & $28(19)^{\star *}$ & $3(5)$ & $\begin{array}{l}24 \\
(15)\end{array}$ & $7(17)$ & $20(18)$ & $11(12)$ & $\underset{7,72^{\star *}}{17,5} \pm$ & $\begin{array}{c}1,80 \pm \\
1,16^{*}\end{array}$ & $31(18)$ \\
\hline Fácil & $120(81)$ & \begin{tabular}{|l}
57 \\
$(95)$
\end{tabular} & $\begin{array}{l}142 \\
(85)\end{array}$ & $35(83)$ & $93(82)$ & $84(88)$ & $\underset{6,12}{21,6} \pm$ & $\begin{array}{c}1,45 \\
1,13\end{array} \pm$ & $177(82)$ \\
\hline \multicolumn{10}{|c|}{ Agarrar objetos com as mãos } \\
\hline Difícil & $15(10)$ & $4(7)$ & $12(7)$ & $7(17)$ & $14(12)$ & $5(5)^{\star \star}$ & $\begin{array}{c}14,4 \pm \\
6,82^{\star *}\end{array}$ & $\begin{array}{c}1,89 \pm \\
1,28^{*}\end{array}$ & $19(11)$ \\
\hline Fácil & $133(90)$ & $\begin{array}{l}56 \\
(93)\end{array}$ & $\begin{array}{l}154 \\
(93)\end{array}$ & $35(83)$ & $99(88)$ & $90(95)$ & $\underset{6,14}{21,7} \pm$ & $\underset{1,12}{1,47} \pm$ & $189(89)$ \\
\hline
\end{tabular}

Notas: DP = Desvio-padrão; GDS = Geriatric Depression Scale; MEEM; § Teste de Qui-quadrado, £ Análise de variância, * p entre 0,1 e $0,05, * * p<0,05$. 
A Tabela 3 mostra as frequências dos fatores sociodemográficos e as médias dos fatores clínicos (MEEM e GDS) entre o grau de facilidade e dificuldade no desempenho das atividades cotidianas. Observa-se que tanto a maioria dos homens quanto das mulheres relataram maior facilidade em realizar as atividades cotidianas. Contudo, os percentuais de facilidade em realizar as atividades pelos homens ainda foram maiores e significativos. Quanto a relação com a faixa etária foi observado que ambos os grupos mais jovens ou mais longevos relataram maiores percentuais de facilidade para realizar tais atividades. Contudo os indivíduos mais jovens apresentaram maior facilidade de realizar as atividades quando comparados com os de mais de 95 anos. Com relação com os anos de estudo observou-se que ambos os grupos de escolaridade apresentaram facilidade de realizar as atividades cotidianas, contudo indivíduos com mais de cinco anos de estudo apresentavam maior facilidade de realizar as atividades. Observou-se que o relato de facilidade de realizar as atividades cotidianas estava relacionado com maiores médias no MEEM e menores médias no GDS, sendo essas relações significativas. A atividade de transferir-se entre assentos foi significativa com sexo e anos de estudo. Em geral se observa que os participantes tinham maior facilidade de realizar as atividades cotidianas do que as atividades funcionais.

Tabela 3 - Atividades cotidianas relacionadas com o escore total do MEEM, Porto Alegre/RS, Brasil, $2019(n=208)$.

\begin{tabular}{|c|c|c|c|c|c|c|c|c|c|}
\hline \multirow{2}{*}{$\begin{array}{c}\text { Variá- } \\
\text { veis }\end{array}$} & \multicolumn{2}{|c|}{ Sexo§ } & \multicolumn{2}{|c|}{ Faixa Etária§ } & \multicolumn{2}{|c|}{ Anos de estudo§ } & \multirow{2}{*}{$\begin{array}{c}\text { MEEM£ } \\
\begin{array}{c}\text { Média } \pm \\
\text { DP }\end{array}\end{array}$} & \multirow{2}{*}{$\begin{array}{c}\text { GDS£ } \\
\text { Média } \\
\pm \text { DP }\end{array}$} & \multirow{2}{*}{$\frac{\text { Total }}{\mathrm{n}(\%)}$} \\
\hline & F n (\%) & $\begin{array}{c}M \text { n( } \\
\%)\end{array}$ & $<95$ & $\geq 95$ & $<5$ & $\geq 5$ & & & \\
\hline \multicolumn{10}{|c|}{ Transferir-se entre assentos } \\
\hline Difícil & $43(29)$ & $\begin{array}{l}4 \\
(7)^{\star \star}\end{array}$ & $\begin{array}{l}34 \\
(21)\end{array}$ & $13(31)$ & $35(31)$ & $12(13)^{\star \star}$ & $\begin{array}{c}16,08 \pm \\
6,56^{\star \star}\end{array}$ & $\begin{array}{c}1,89 \pm \\
1,22^{\star *}\end{array}$ & $47(23)$ \\
\hline Fácil & $105(71)$ & $\begin{array}{l}5 \\
(93)\end{array}$ & $\begin{array}{lll}1 & 3 & 2 \\
(79) & \end{array}$ & $29(69)$ & $78(69)$ & $83(87)$ & $\begin{array}{c}22,44 \\
5,80\end{array}$ & $\underset{1,10}{1,39} \pm$ & 161(77) \\
\hline \multicolumn{10}{|c|}{ Tomar banho sozinho } \\
\hline Difícil & $48(32)$ & \begin{tabular}{|l|}
11 \\
$(18)$
\end{tabular} & $\begin{array}{l}39 \\
(24)\end{array}$ & $20(48)$ & $40(35)$ & $19(20)$ & $\begin{array}{c}16,16 \pm \\
7,06^{\star \star}\end{array}$ & $\begin{array}{c}1,91 \pm 1 \\
, 20^{\star *}\end{array}$ & $59(28)$ \\
\hline Fácil & $100(68)$ & $\begin{array}{l}49 \\
(82)\end{array}$ & $\begin{array}{l}127 \\
(76)\end{array}$ & $22(52)$ & $73(65)$ & $76(80)$ & $\begin{array}{c}22,9 \quad 1 \pm \\
5,21\end{array}$ & $\underset{1,34}{1,08} \pm$ & $149(72)$ \\
\hline \multicolumn{10}{|c|}{ Vestir-se sozinho } \\
\hline Difícil & $41(28)$ & $\begin{array}{l}9 \\
(15)\end{array}$ & $\begin{array}{l}31 \\
(19)\end{array}$ & $19(45)$ & $37(33)$ & $13(14)$ & $\begin{array}{c}15,92 \pm \\
7,21^{\star *}\end{array}$ & $\begin{array}{c}1,98 \pm \\
1,09^{\star \star}\end{array}$ & $50(24)$ \\
\hline Fácil & $107(72)$ & $\begin{array}{l}51 \\
(85)\end{array}$ & $\begin{array}{l}135 \\
(81)\end{array}$ & $23(55)$ & $76(67)$ & $82(86)$ & $\underset{5,40}{22,61} \pm$ & $\underset{1,12}{1,36} \pm$ & $158(76)$ \\
\hline
\end{tabular}




\begin{tabular}{|c|c|c|c|c|c|c|c|c|c|}
\hline \multicolumn{10}{|c|}{ Alimentar-se sozinho } \\
\hline Difícil & $13(9)$ & $1(2)$ & $9(5)$ & $5(12)$ & $11(10)$ & $3(3)$ & $\begin{array}{c}11,6 \pm \\
7,13^{\star \star}\end{array}$ & $\begin{array}{c}2,42 \pm \\
1,22^{\star *}\end{array}$ & $14(7)$ \\
\hline Fácil & $135(91)$ & $\begin{array}{l}59 \\
(98)\end{array}$ & $\begin{array}{l}157 \\
(95)\end{array}$ & $37(88)$ & $\begin{array}{c}10^{0} 2 \\
(90)\end{array}$ & $92(97)$ & $\begin{array}{c}21, \quad 7 \pm \\
5,96\end{array}$ & $\underset{1,11}{1,44} \pm$ & $194(93)$ \\
\hline \multicolumn{10}{|c|}{ Usar o banheiro } \\
\hline Difícil & $27(18)$ & $2(3)$ & $\begin{array}{l}16 \\
(10)\end{array}$ & $13(31)$ & $20(18)$ & $9(9)$ & $\begin{array}{c}14,9 \pm \\
7,02^{\star *}\end{array}$ & $\begin{array}{c}2,17 \pm \\
1,22^{\star \star}\end{array}$ & $29(14)$ \\
\hline Fácil & $121(82)$ & $\begin{array}{l}58 \\
(97)\end{array}$ & $\begin{array}{l}150 \\
(90)\end{array}$ & $29(69)$ & $93(82)$ & $86(91)$ & $\underset{5,90}{22,0} \pm$ & $\underset{1,09}{1,40} \pm$ & $179(86)$ \\
\hline
\end{tabular}

Notas: DP = Desvio-padrão; GDS= Geriatric Depression Scale; MEEM= Mini Exame do Estado Mental; § Teste de qui-quadrado, £ Análise de variância, * p entre 0,1 e $0,05, * * \mathrm{p}<0,05$

Nas regressões univariadas ajustadas pelos anos de estudo observa-se que a presença de anos de estudo em cada modelo independente diminuía o efeito das variáveis sociodemográficas, atividades funcionais e cotidianas sobre os níveis do escore do MEEM. O mesmo se observa nos modelos univariados ajustados com a GDS e seu modelo é muito próximo do efeito do ajuste pelos anos de estudo. $\mathrm{O}$ mesmo efeito foi observado em relação ao ajuste para sexo e ajuste por faixa etária, mostrando que o efeito da quantidade de anos de estudo, da pontuação GDS, sexo e faixa etária é muito similar uma em relação à outra, nessa relação entre a funcionalidade e as atividades cotidianas com o escore do MEEM.

A atividade de alimentar-se sozinho, usar o banheiro, agarrar objetos com as mãos, foram as atividades com o maior coeficiente relacionado ao escore do MEEM, onde, por exemplo, referir ter facilidade em alimentar-se sozinho, estaria relacionado em um aumento de dez pontos no escore do MEEM. 
abela 4 - Modelos de regressão linear para a predição de MEEM univariados e ajustados para Anos de estudo, GDS, Sexo e Faixa etária, Porto Alegre/RS, Brasil, $2019(n=208)$.

\begin{tabular}{|c|c|c|c|c|c|c|c|c|c|c|}
\hline $\begin{array}{l}\text { Regres- } \\
\text { são para } \\
\text { MEEM } \\
\text { univaria- } \\
\text { das }\end{array}$ & $\begin{array}{r}\text { ME } \\
\text { (Não a }\end{array}$ & $\begin{array}{l}\text { EM } \\
\text { ustado) }\end{array}$ & $\begin{array}{r}\text { Ajustac } \\
\text { Anos } \\
\text { tu }\end{array}$ & $\begin{array}{l}\text { o para } \\
\text { le es- } \\
\text { lo }\end{array}$ & $\begin{array}{r}\text { Ajusta } \\
\mathrm{G}\end{array}$ & $\begin{array}{l}\text { lo para } \\
\text { S }\end{array}$ & $\begin{array}{r}\text { Ajusta } \\
\mathrm{Se}\end{array}$ & $\begin{array}{l}\text { do para } \\
\text { xo }\end{array}$ & $\begin{array}{r}\text { Ajusta } \\
\text { Faixa }\end{array}$ & $\begin{array}{l}\text { do para } \\
\text { etária }\end{array}$ \\
\hline $\begin{array}{l}\text { Anos de } \\
\text { estudo }(\geq \\
5 /<5)\end{array}$ & 0,266 & $\begin{array}{l}< \\
0,001\end{array}$ & - & - & 0,232 & $\begin{array}{l}< \\
0,001\end{array}$ & 0,229 & 0,001 & 0,250 & $<0,001$ \\
\hline GDS & $-1,351$ & 0,001 & $-1,108$ & 0,004 & - & - & $-1,236$ & 0,001 & $-1,227$ & 0,001 \\
\hline $\begin{array}{l}S \text { e } x \quad o \\
\text { (masculi- } \\
\text { no/femini- } \\
\text { no) }\end{array}$ & 3,273 & 0,001 & 2,545 & 0,010 & 2,966 & 0,002 & - & - & 2,933 & 0,002 \\
\hline $\begin{array}{l}\text { Faixa } \\
\text { etária }(\geq \\
95 /<95)\end{array}$ & $-3,884$ & 0,001 & $-3,583$ & 0,001 & $-3,533$ & 0,001 & $-3,524$ & 0,001 & - & - \\
\hline $\begin{array}{l}\text { Desem- } \\
\text { penho no } \\
\text { Caminhar } \\
400 \mathrm{~m}\end{array}$ & 3,645 & $\begin{array}{l}< \\
0,001\end{array}$ & 3,083 & 0,001 & 3,004 & 0,001 & 3,197 & $\begin{array}{l}< \\
0,001\end{array}$ & 3,092 & $<0,001$ \\
\hline $\begin{array}{l}\text { Subir dez } \\
\text { degraus }\end{array}$ & 4,685 & $\begin{array}{l}< \\
0,001\end{array}$ & 4,026 & $\begin{array}{l}< \\
0,001\end{array}$ & 4,210 & $\begin{array}{l}< \\
0,001\end{array}$ & 4,287 & $\begin{array}{l}< \\
0,001\end{array}$ & 4,258 & $<0,001$ \\
\hline $\begin{array}{l}\text { Levantar } \\
\text { da cadeira } \\
\text { sem apoio }\end{array}$ & 3,811 & $\begin{array}{l}< \\
0,001\end{array}$ & 3,528 & $\begin{array}{l}< \\
0,001\end{array}$ & 3,435 & $\begin{array}{l}< \\
0,001\end{array}$ & 3,482 & $\begin{array}{l}< \\
0,001\end{array}$ & 3,194 & $<0,001$ \\
\hline $\begin{array}{l}\text { Carregar } \\
\text { cinco } \\
\text { quilos }\end{array}$ & 4,314 & $\begin{array}{l}< \\
0,001\end{array}$ & 3,678 & $\begin{array}{l}< \\
0,001\end{array}$ & 3,737 & $\begin{array}{l}< \\
0,001\end{array}$ & 3,743 & $\begin{array}{l}< \\
0,001\end{array}$ & 3,871 & $<0,001$ \\
\hline $\begin{array}{l}\text { Agachar- } \\
\text {-se }\end{array}$ & 3,196 & 0,001 & 3,033 & 0,001 & 2,574 & 0,005 & 2,853 & 0,001 & 3,102 & $<0,001$ \\
\hline $\begin{array}{l}\text { Levantar } \\
\text { os braços } \\
\text { acima do } \\
\text { nível da } \\
\text { cabeça }\end{array}$ & 4,100 & 0,001 & 3,529 & 0,004 & 3,672 & 0,003 & 3,471 & 0,006 & 3,993 & 0,001 \\
\hline $\begin{array}{l}\text { Agarrar } \\
\text { objetos } \\
\text { com as } \\
\text { mãos }\end{array}$ & 7,246 & $\begin{array}{l}< \\
0,001\end{array}$ & 6,696 & $\begin{array}{l}< \\
0,001\end{array}$ & 6,750 & $\begin{array}{l}< \\
0,001\end{array}$ & 6,986 & $\begin{array}{l}< \\
0,001\end{array}$ & 6,649 & $\begin{array}{l}< \\
0,001\end{array}$ \\
\hline $\begin{array}{l}\text { Transferir- } \\
\text {-se entre } \\
\text { assentos }\end{array}$ & 6,356 & $\begin{array}{l}< \\
0,001\end{array}$ & 5,792 & $\begin{array}{l}< \\
0,001\end{array}$ & 5,879 & $\begin{array}{l}< \\
0,001\end{array}$ & 5,840 & $\begin{array}{l}< \\
0,001\end{array}$ & 6,042 & $<0,001$ \\
\hline
\end{tabular}




\begin{tabular}{|l|l|l|l|l|l|l|l|l|l|l|}
\hline $\begin{array}{l}\text { Tomar } \\
\text { banho } \\
\text { sozinho }\end{array}$ & 6,750 & $\begin{array}{l}< \\
0,001\end{array}$ & 6,252 & $\begin{array}{l}< \\
0,001\end{array}$ & 6,299 & $\begin{array}{l}< \\
0,001\end{array}$ & 6,413 & $<$ & 6,297 & $<$ \\
\hline $\begin{array}{l}\text { Vestir-se } \\
\text { sozinho }\end{array}$ & 6,694 & $\begin{array}{l}< \\
0,001\end{array}$ & 6,189 & $\begin{array}{l}< \\
0,001\end{array}$ & 6,189 & $\begin{array}{l}< \\
0,001\end{array}$ & 6,342 & $<$ & 6,167 & $<$ \\
\hline $\begin{array}{l}\text { Alimentar- } \\
\text {-se sozi- } \\
\text { nho }\end{array}$ & 10,038 & $<$ & 9,532 & $<$ & 9,132 & $<$ & 9,432 & $<$ \\
0,001 & 0,001 & 0,001 & 0,001 & 9,494 & $<$ \\
\hline $\begin{array}{l}\text { Usar o } \\
\text { banheiro }\end{array}$ & 7,098 & $\begin{array}{l}< \\
0,001\end{array}$ & 6,641 & $\begin{array}{l}< \\
0,001\end{array}$ & 6,406 & $\begin{array}{l}< \\
0,001\end{array}$ & 6,511 & $<$ & 6,375 & $<$ \\
\hline
\end{tabular}

Notas: GDS= Geriatric Depression Scale.

\section{Discussão}

Este estudo buscou identificar quais atividades funcionais e cotidianas estão relacionadas com o desempenho cognitivo de longevos e o impacto das mesmas sobre a pontuação geral obtida no MEEM, ajustando por determinantes cognitivos conhecidos, como sexo, idade, anos de estudo e sintomas depressivos. Observamos que o grau de facilidade do desempenho tanto de atividades funcionais quanto o de cotidianas foram significativamente relacionados ao desempenho cognitivo dos longevos avaliados.

Entre os longevos analisados encontramos diferenças significativas no desempenho cognitivo, variáveis sociodemográficas (sexo, faixa-etária, escolaridade) e sintomas depressivos. Estudo realizado com longevos em dois municípios encontrou resultados parecidos: idade inversamente proporcional ao MEEM e homens apresentando um maior escore comparado às mulheres (ROSSET et al., 2011). A relação com a escolaridade também foi observada pelo estudo de Macuco (2011) em idosos mais jovens que o da presente pesquisa.
Quanto menor a escolaridade menor foi o desempenho no MEEM. O presente trabalho conclui que essas observações também são válidas para pessoas com 90 anos ou mais, sugerindo que a reserva cognitiva é importante nessa faixa etária.

As diferenças no desempenho cognitivo, entre as características sociodemográficas e clínicas, motivou a realização de uma análise múltipla, para observar se essas características seriam fatores determinantes na relação entre o desempenho cognitivo e o grau de facilidade no desempenho das atividades funcionais e cognitivas. Para tanto, incluímos nos modelos de regressão linear as variáveis: anos de estudo, faixa etária, sexo e GDS, individualmente. A presença das variáveis sociodemográficas (sexo, faixa etária e escolaridade) nos modelos de regressão linear, reduziram o efeito das atividades funcionais e cotidianas sobre os níveis do escore do MEEM. No entanto, todas as relações se mantiveram significativas, permitindo a conclusão que a relação entre o desempenho cognitivo e o grau de facilidade na execução de atividades funcionais e cotidianas independem dos 
fatores sociodemográficos e clínicos. Desta forma o presente trabalho apresenta uma importante observação, que talvez a manutenção das atividades funcionais e cotidianas possam exercer um papel significativamente importante na manutenção do desempenho cognitivo. Mesmo com uma capacidade intrínseca diminuída, longevos podem manter habilidades funcionais através de intervenções adaptativas.

Uma das adaptações possíveis de realizar para facilitar a realização das atividades é transformar o ambiente da casa propício e satisfatório para o idoso, oferecendo segurança para realizar as atividades de forma funcional, com estímulos e controle pessoal, facilitando a interação social (MESSIAS; NEVES, 2009).

Visando as dificuldades de realizar as atividades cotidianas e funcionais, existem alguns dispositivos que podem facilitar as atividades, que é a tecnologia assistiva, a qual se refere a diversos tipos de dispositivos de auxílios estratégicos, que visam reduzir o impacto da disfunção física, ao prover uma conexão entre as limitações funcionais do indivíduo e as demandas do meio físico. Vários tipos de tecnologias têm sido propostos e implementados para atender aos idosos fragilizados e dependentes que tem necessidade de manter sua capacidade funcional, autonomia e qualidade de vida (ANDRADE; PEREIRA, 2009).

Um estudo realizado com dez idosos no município de São Paulo, após algumas adaptações na casa dos idosos, conclui que as adaptações e tecnologia assisti- va aumentam a participação do idoso na rotina de atividades do domicílio, promovendo um maior envolvimento do mesmo com a família e ampliando o repertório ocupacional, resgatando atividades significativas desenvolvidas no passado e possibilitando a inclusão de novas atividades e interesses na rotina (SILVA et al., 2011). O estudo, no entanto, não verificou se havia ou não ocorrido uma melhora no desempenho cognitivo dos participantes, embora essa conclusão parece ser implícita nos resultados.

Em um estudo realizado com 819 idosos com o objetivo de avaliar o impacto das atividades avançadas de vida diária (AAVD) na incidência de declínio cognitivo mostram que o desempenho de AAVD foi um fator protetor para o desenvolvimento de declínio cognitivo, demonstrando a necessidade de diversidade de estímulos e de altos níveis de envolvimento em atividades para um melhor desempenho cognitivo (DIAS et al., 2015).

Uma pesquisa que teve como objetivo relacionar o impacto do comprometimento cognitivo nas atividades da vida diária (AVD) para ser utilizado como critério importante para diferenciar entre comprometimento cognitivo leve (CCL) e demência, concluiu que houve evidência de que pacientes com declínio cognitivo leve apresentariam menores escores no MEEM e no desempenho de atividades complexas, especialmente com relação a tarefas que envolviam memória ou raciocínio complexo (PERNECZKY et al., 2006). Sendo que no presente estudo chegamos também a conclusão que 
atividades que têm maior complexidade são as que os idosos apresentam maior dificuldade em realizar. Sendo um importante fator para junto com as avaliações cognitivas, realizar a avaliação também a realização de atividades mais simples e outras mais complexas.

Em um estudo destacou a importância de se colocar em exposição a ambientes complexos e estimulantes ao longo da vida, como realizam de atividades laborais mais complexas, alto nível de escolaridade, engajamento de atividades de lazer e envolvimentos de atividades que requerem mais o uso cognitivo para serem realizadas, esses fatores contribuem com a construção, formação e manutenção de melhores níveis de reserva cognitiva, proporcionando maior resistência aos danos cerebrais e poderiam postergar o aparecimento de possíveis déficits cognitivos do envelhecimento (SPOSITO et al., 2016).

A manutenção da cognição é de relevância para a promoção da independência e autonomia do idoso e a sua estimulação pode prevenir ou mesmo retardar o processo de degeneração do cérebro. Por isso, a atividades de estimulação cognitiva são importantes para idosos, para evitar ou retardar as doenças e também privilegiar o envelhecimento sadio. Desta forma, o envolvimento em atividades de estimulação cognitiva e um estilo de vida mais ativo e engajado socialmente têm um efeito positivo na cognição e na possibilidade de prevenir declínios cognitivos e depressão (CASEMIRO et al., 2016).
Estimular a manutenção da funcionalidade é de fundamental importância pois, mesmo estudos longitudinais mais antigos, encontraram que as funções cognitivas predizem mortalidade, num seguimento de três anos, independentemente da idade, sexo, nível educacional e sintomas depressivos (SMITS et al., 1999). E uma pesquisa mais recente indica que a queixa funcional, em indivíduos sem comprometimento cognitivo, pode ser preditora de declínio cognitivo leve em idosos, onde se observou que os indivíduos que apresentaram declínio cognitivo leve o demonstraram em 3 anos de seguimento médio, numa amostra com média etária de 75,5 anos e média de anos de estudo maior que a investigada neste estudo (13, 9 \pm 3,7 anos) (FARIAS et al., 2017).

Uma limitação da pesquisa foi que estudos transversais não permitem estabelecer se níveis funcionais prévios mais altos possam ter favorecido a manutenção da capacidade cognitiva nesses nonagenários, compensando a baixa escolaridade, frequente entre esses longevos. Por isso, há a necessidade de novos estudos, de caráter longitudinal para melhorar esse entendimento.

\section{Conclusões}

Concluímos que o relato de facilidade em desempenhar as atividades cotidianas e funcionais analisadas foram relacionadas a um melhor desempenho cognitivo e que essa associação independe de fatores sociodemográficos e clínicos, como sexo, idade, escolaridade 
e sintomas depressivos. As atividades cotidianas tiveram um efeito maior sobre o MEEM do que as funcionais no teste de regressão linear quando se observa maiores coeficientes de regressão nesse tipo de atividades, mais relacionadas ao autocuidado. Os achados também sugerem que adaptações ao meio ambiente favorecendo a realização dessas atividades podem ser também benéficas à manutenção do desempenho cognitivo.

\section{Functional and daily living activities related to the cognitive performance of nonagenarians}

\section{Abstract}

The objective of the study was to investigate the relationship between cognition and the ease to perform daily and functional activities in nonagenarians and the effect of investigated activities on cognition, adjusting for sex, age, schooling and depressive symptoms. This is a cross-sectional and analytical study. We selected community dwelling nonagenarians from Porto Alegre, RS in 2016 through cluster sampling. The variables of the study were the cognition (Mental State Mini Exam - MMSE), socio-demographic variables (gender, schooling and age group) and depressive symptoms (Geriatric Depression Scale -GDS) and the ease of performing 12 functional and daily living activities. The ease of performing each activity had its relation with the MEEM tested by the chi-square test, ANO$\mathrm{VA}$ and simple linear regression adjusted for sociodemographic and clinical characteristics. There were 208 nonagenarians, $71 \%$ women. The ease to perform functio- nal and daily living activities was significantly related to higher scores in the MMSE as in the simple as in the adjusted analyses. Males, more than 5 years of study, lower depressive symptoms and less than 95 years were related to greater reports of ease of performing daily living and functional activities. The daily living activities related to the self-care achieved the highest coefficient of regression to MEEM scores. The findings suggest that the ease to perform such activities is associated to higher cognitive scores in nonagenarians, independently of schooling, depressive symptoms, gender and age group.

Keywords: Longevity. Aging. Dementia. Activities of Daily Living. Cognition.

\section{Referências}

ALMEIDA, M. S. C. Efetividade da escala de depressão geriátrica de cinco itens em população idosa da comunidade. 133 f. 2010. Tese (Doutorado) - Pontifícia Universidade Católica do Rio Grande do Sul. Faculdade de Medicina. Programa de Pós-Graduação em Medicina e Ciências da Saúde. Porto Alegre: PUCRS, 2010.

ANDRADE, V. S.; PEREIRA, L. S. M. Influence of assistive technology on the functional performance and quality of life of community-dwelling frail elderly people: a bibliographic review. Revista Brasileira de Geriatria e Gerontologia, Rio de Janeiro, v. 12, n. 1, p. 113-122, 2009.

CASEMIRO, F. G. et al. Impact of cognitive stimulation on depression, anxiety, cognition and functional capacity among adults and elderly participants of an open university for senior citizens. Revista Brasileira de Geriatria e Gerontologia, Rio de Janeiro, v. 19, n. 4, p. 683-694, 2016.

CASTRO-COSTA, E. et al. Trajectories of cognitive decline over 10 years in a Brazilian elderly population: the Bambuí Cohort Study 
of Aging. Caderno de Saúde Pública, Rio de Janeiro, v. 27, n. 3, p.345-350, 2011.

CHAVES, A. S. et al. Association between cognitive decline and quality of life of hypertensive elderly individuals. Revista Brasileira de Geriatria e Gerontologia, Rio de Janeiro, v. 18, n. 3, p. 545-556, 2015.

CÔRRO, H. S. F. L. Centenários: um modelo de longevidade. 2016. 75f. Dissertação (Mestrado Integrado em Medicina) - Universidade de Coimbra, Faculdade de Medicina, Coimbra, Portugal, 2016. Disponível em: <https://estudogeral.sib.uc.pt/ bitstream/10316/36188/1/A\%20TESE\%20 Helena\%20Côrro.pdf>. Acesso em: 1 nov. 2017.

DIAS, E. G. et al. Atividades avançadas de vida diária e incidência de declínio cognitivo em idosos: Estudo SABE. Cadernos de Saúde Pública, São Paulo, v. 31, n. 8, p. 1623-1635, 2015.

FARIAS, S. T. et al. Early functional limitations in cognitively normal older adults predict diagnostic conversion to mild cognitive impairment. Journal of the American Geriatrics Society, v. 65, n. 6, p. 1152-1158, 2017.

FECHINE, B. R. A.; TROMPIERI, N. O processo de envelhecimento: as principais alterações que acontecem com o idoso com o passar dos anos. Interscienceplace International Scientific Journal, Campos de Goytacazes, v. 20, n. 1, p. 106-194, 2012.

FOLSTEIN, M. F.; FOLSTEIN, S. E.; MCHUGH, P. R. Mini Mental State: A practical method for grading the cognitive state of patients for the clinical. Journal of Psychiatric Research, v. 12, n. 3, p. 189-198, 1975.

GOYANNA, N. F. et al. Elderly with Alzheimer's disease: how they live and notice the attention in the health strategy of the family. Revista de Pesquisa: Cuidado é Fundamenta Online, Rio de janeiro, v. 9, n. 2, p. 379-386, 2017.

LIVINGSTON, G. et al. Dementia prevention, intervention, and care. The Lancet,
2017. 62p. Disponível em: <http://www.thelancet.com/pdfs/journals/lancet/PIIS01406736(17)31363-6.pdf>. Acesso em: 6 nov. 2017.

MACUCO, C. R. M. Desempenho no MEEM em idosos frágeis e pré-frágeis e não frágeis residentes na comunidade: dados do estudo FIBRA em Ermelino Matarazzo São Paulo. 2011. 241 f. Dissertação (Mestrado em Gerontologia) - Universidade Estadual de Campinas, Faculdade de Ciências Médicas, Campinas, 2011.

MESSIAS, M. G.; NEVES, R. F. A influência de fatores comportamentais e ambientais domésticos nas quedas em idosos. Revista Brasileira de Geriatria e Gerontologia, Rio de Janeiro, v. 12, n. 2, p. 275-282, 2009.

NASCIMENTO, R. A. S. A. D. et al. Prevalence and factors associated with the decline in the elderly with cognitive low economic condition: MONIDI study. Jornal Brasileiro de Psiquiatria, Rio de Janeiro, v. 64, n. 3, p. 187-192, 2015.

OBSERVAPOA - Observatório da Cidade de Porto Alegre. Porto Alegre em mapas: territorialidades: regiões OP [Internet]. Porto Alegre; 1997.Disponível em: <http://www. observapoa.com.br/default.php?p_secao $=46>$. Acesso em: 31 ago. 2019.

PERNECZKY, R. et al. Complex activities of daily living in mild cognitive impairment: conceptual and diagnostic issues. Age Ageing , v. 35 , p. $240-245,2006$.

ROSSET, I. et al. Diferenciais socioeconômicos e de saúde entre duas comunidades de idosos longevos. Revista de Saúde Pública, São Paulo, v. 45, n. 2, p. 391-400, 2011.

SILVA, A. P. L. L. D. et al. Effectiveness of the environmental adaptation: the perception of caregivers. Revista Equilíbrio Corporal e Saúde, São Paulo, v. 3, n. 1, p. 25-40, 2011.

SIMONSICK, E. M. et al. Measuring higher level physical function in well-functioning older adults: expanding familiar approaches in the Health ABC study. The journals of 
gerontology. Series A, Biological Sciences and Medical Sciences, v. 56, n. 10, p. M644649, 2001.

SMITS, C. H. M. et al. Cognitive functioning and health as determinants of mortality in an older population. American Journal of Epidemiology, v. 150, n. 9, p. 978-986, 1999.

SPOSITO, G.; NERI, A. L.; YASSUDA, M. S. Advanced activities of daily living (AADLs) and cognitive performance in community-dwelling elderly persons: Data from the FIBRA Study - UNICAMP. Revista Brasileira de Geriatria e Gerontologia, Rio de Janeiro, v. 19, n. 1, p. 7-20, 2016. 